\title{
Liftings of formal groups and the Artinian completion of $v_{n}^{-1} \mathrm{BP}$
}

\author{
BY ANDREW BAKER \\ Manchester University, Manchester M13 9PL, England \\ AND URS WÜRGLER \\ Universität Bern, Bern, CH3012, Switzerland \\ (Received 1 October 1988; revised 17 February 1989) \\ Introduction
}

Let $v_{n}^{-1} \mathrm{BP}$ denote the localization at $v_{n}$ of the Brown-Peterson spectrum (associated to the prime $p$ ). There is a related ring spectrum $E(n)$ with homotopy ring

$$
\pi_{*}(E(n))=\mathbb{Z}_{(p)}\left[v_{1}, v_{2}, \ldots, v_{n}, v_{n}^{-1}\right]
$$

(as a quotient ring of $\left.\pi_{*}\left(v_{n}^{-1} \mathrm{BP}\right)\right)$; in fact the cohomology theory $E(n)^{*}()$ is determined via a Conner-Floyd type isomorphism from $v_{n}^{-1} \mathrm{BP} *()$ on finite complexes, and moreover $E(n)$ and $v_{n}^{-1} \mathrm{BP}$ are in the same Bousfield class (see $[2,14]$ ). Although it is known (essentially from [17]) that $v_{n}^{-1} \mathrm{BP}$ cannot be a product of suspensions of $E(n)$ in a multiplicative sense, D. Ravenel conjectured that such a splitting might occur after suitable completion of these spectra (see the introduction to [14]). This question was the original motivation of the present paper; however in proving Ravenel's conjecture we were naturally led to the consideration of some fundamental results in the theory of liftings of formal group laws and change of ring' results for Ext groups occurring in connection with the work of $[10,11,12]$.

In order to prove our topological results we first need to derive some algebraic facts. In Section 1 we consider the problem of lifting $p$-typical formal group laws and their strict isomorphisms from an $\mathbb{F}_{p}$-algebra $\mathbf{k}$ to an Artinian local ring $A$ with residue field $\mathbf{k}=A / \mathbf{m}$. We construct an idempotent functor

$$
\text { e : } \operatorname{lift}_{n}(A) \rightarrow \operatorname{lift}_{n}(A)
$$

on the groupoid of all such lifts having height $n(>0)$ which assigns to $F \in \operatorname{Obj} \operatorname{lift}_{n}(A)$ a new group law $F^{\prime \prime}$ with $p$-series of form

$$
[p]_{F^{\prime}} X=\sum_{0 \leqslant i \leqslant n}^{F^{\prime}}\left(a_{i} X^{p^{i}}\right)
$$

Then the image of e turns out to be equivalent to the quotient category of lift ${ }_{n}(A)$ by *-isomorphisms (see [8]). Indeed we reprove Lubin and Tate's results on such liftings in the precise form required for Morava's applications (see [10]). Our main technical ingredient is an infinite-dimensional version of Hensel's Lemma, which may be of independent interest.

In Section 2 we introduce the Artinian completions $\widehat{v_{n}^{-1} \mathrm{BP}}, \widehat{E(n)}$ of the ring spectra $v_{n}^{-1} \mathrm{BP}, E(n)$ of [14]. Using algebraic results from Section 1 we are then able to construct an idempotent morphism of ring spectra

$$
\Phi: \widehat{v_{n}^{-1} \mathrm{BP}} \rightarrow \widehat{v_{n}^{-1} \mathrm{BP}}
$$


factoring through the canonical morphism

$$
\widehat{v_{n}^{-1} \mathrm{BP}} \rightarrow \widehat{E(n)}
$$

and inducing a splitting (of $\widehat{E(n)}$-module spectra)

$$
\widehat{v_{n}^{-1} \mathrm{BP}} \simeq \prod_{\alpha} \Sigma^{\sigma(\alpha)} \widehat{E(n)}
$$

In Section 3 we apply results of Section 1 to give new and conceptual proofs of some change of rings isomorphisms from [11].

Finally in Section 4 we place our work in the context of [2] and [3] by showing that our Artinian completions $\widehat{v_{n}^{-1} \mathrm{BP}}, \widehat{E(n)}$ are $K(n)$ local ; indeed we show that the natural map $E(n) \rightarrow \widehat{E(n)}$ is a $K(n)$ localization, thus generalizing a result of $\mathrm{A}$. K. Bousfield for the case $n=1$. We make use of a construction similar to Sullivan's profinite completion [16], and also require recent work of Hopkins, Devinatz and Smith [3].

\section{Canonical lifting of Lubin-Tate laws}

In this section we describe our algebraic results. Our viewpoint is essentially that of Morava (see [13, 10]) and is related to [8]. However, as explained in the Introduction, we give new proofs and accounts of much of this material.

Let $p>0$ be a prime. There is a universal (graded) ring for $p$-typical formal group laws over graded $\mathbb{Z}_{(p)}$-algebras

$$
V_{*}=\mathbb{Z}_{(p)}\left[v_{i}: i \geqslant 1\right] \quad\left(v_{0}=p\right),
$$

where the $v_{i}$ denote the Araki generators defined by

$$
[p]_{F^{v}} X=\sum_{0 \leqslant i}^{F^{v}}\left(v_{i} X^{p^{i}}\right)
$$

and where $F^{V}$ is the universal $p$-typical group law over $V$. Let $p$-FGL $(R)$ denote the groupoid of strict isomorphisms of $p$-typical group laws over $R$. Let $\Gamma_{*}=V_{*}\left[t_{i}: i \geqslant 1\right]$ (with $t_{0}=1$ ). Then we have for any $\mathbb{Z}_{(p)}$ algebra $R$ the following result of [6]:

Proposition 1.2. The groupoid valued functor $p$-FGL is (co)represented by $\left(V_{*}, \Gamma_{*}\right)$; i.e. there are natural isomorphisms

$$
p-\mathbf{F G L}(R) \cong \operatorname{Alg}_{\mathbf{z}_{(p)}}\left(\Gamma_{*}, R\right), \quad \operatorname{Obj} p-\mathbf{F G L}(\mathbf{R}) \cong \mathbf{A l g}_{\mathbb{Z}_{(p)}}\left(V_{*}, R\right) .
$$

Here the groupoid structure on $p$-FGL turns $\left(V_{*}, \Gamma_{*}\right)$ into a Hopf algebroid with structure maps

$$
V_{*} \stackrel{\eta_{L}}{\rightarrow} \underset{\eta_{R}}{\rightarrow} \Gamma_{*} \stackrel{e}{\rightarrow} V_{*}
$$

corresponding to the domain, codomain and identity functions. Thus for any strict isomorphism $\phi: F_{1} \cong F_{2}$ we have

$$
\phi^{-1}(X)=\sum_{0 \leqslant i}^{F_{1}}\left(c_{i} X^{p^{i}}\right)
$$

where $\phi$ is classified by the homomorphism $\Phi: \Gamma_{*} \rightarrow R$ extending the classifying map of $F_{1}$ by putting $\Phi\left(t_{i}\right)=c_{i}$.

In fact the pair $\left(V_{*}, \Gamma_{*}\right)$ is equivalent to the pair $\left(\mathrm{BP}_{*}, \mathrm{BP} \mathrm{P}_{*}(\mathrm{BP})\right)$ where $\mathrm{BP}$ is the 
Brown-Peterson spectrum for the prime $p$. This inherits a Hopf algebroid structure from the two topologically induced units, agreeing with that of $\left(V_{*}, \Gamma_{*}\right)$.

We will need to consider a number of related functors. First let us say that a formal group law $F \in \operatorname{Obj} p$-FGL $(R)$ is of co-height $n$ if its $p$-series has form

$$
[p]_{F} X=\underset{0 \leqslant i \leqslant n}{\sum^{F}}\left(c_{i} X^{p^{t}}\right)
$$

with $c_{n} \in R$ a unit. We can then define the groupoid $p$ - $\mathrm{FGL}^{(n)}(R)$ of strict isomorphisms of co-height $n$ objects in $p$-FGL $(R)$. Let $V(n)_{*}=v_{n}^{-1} V_{*}$ and let $E(n)_{*}=V(n)_{*} /\left(v_{n+i}: i \geqslant 1\right)$ with the natural map $\pi: V(n)_{*} \rightarrow E(n)_{*}$. We can define a Hopf algebroid $\left(E(n)_{*}, \Sigma(n)_{*}\right)$ where

$$
\Sigma(n)_{*}=E(n)_{*} \underset{v_{*}}{\otimes} \Gamma_{*} \otimes E(n)_{*}
$$

We have, by an easy argument based on (1.2),

Proposition 1·3. The groupoid valued functor $p$-FGL ${ }^{(n)}$ is (co)represented by $\left(E(n)_{*}, \Sigma(n)_{*}\right)$; i.e. there are natural isomorphisms

$$
p-\mathrm{FGL}^{(n)}(R) \cong \operatorname{Alg}_{\mathbf{z}_{(p)}}\left(\Sigma(n)_{*}, R\right), \quad \operatorname{Obj} p-\mathrm{FGL}^{(n)}(R) \cong \operatorname{Alg}_{\mathbf{z}_{(p)}}\left(E(n)_{*}, R\right) .
$$

Now for any $\mathbb{F}_{p}$-algebra $R$ a height $n$ Lubin-Tate law over $R$ is an $F \in \operatorname{Obj} p$-FGL $(R)$ such that the classifying map $V_{*} \rightarrow R$ factors as

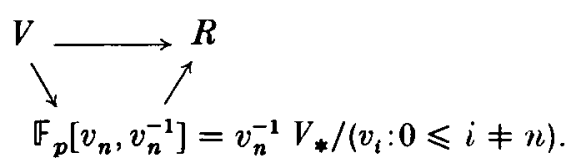

We will only concern ourselves with the case when $n>0$. The full subcategory of $p$-FGL $(R)$ with these as objects will be denoted by $\mathbf{L}-\mathbf{T}_{n}(R)$. Notice that $\mathbf{L}-\mathbf{T}_{n}(R)$ is a subgroupoid of $p$-FGL ${ }^{(n)}(R)$. The following is easily verified and is essentially proved in [13].

Proposition 1.4. $\quad \mathbf{L}_{n} \mathbf{T}_{n}(R) \cong \operatorname{Alg}_{\mathbb{F}_{p}}\left(\mathbb{F}_{p}\left[v_{n}, v_{n}^{-1}\right]\left[t_{i}: i \geqslant 1\right] /\left(t_{i}^{p^{n}}-v_{n}^{p^{t}-1} t_{i}: i \geqslant 1\right), R\right)$. Moreover, there is a natural decomposition

The point here is that

$$
\mathrm{L}_{n} \mathrm{~T}_{n}(R) \cong \underset{F \in \mathrm{Obj}^{2}-\mathrm{T}_{n}(R)}{\amalg} \operatorname{Aut}\left(\boldsymbol{F}^{\prime}\right) .
$$

$$
\mathbb{F}_{p}\left[v_{n}, v_{n}^{-1}\right]\left[t_{i}: i \geqslant 1\right] /\left(t_{i}^{p^{n}}-v_{n}^{p^{i}-1} t_{i}: i \geqslant 1\right)
$$

is a Hopf algebra over $\mathbb{F}_{p}\left[v_{n}, v_{n}^{-1}\right]$.

Now let $\mathrm{Art}_{p}$ denote the category of Artinian local rings with residue field $A / \mathrm{m}$ of characteristic $p$. Then for $F \in \operatorname{Obj} \mathrm{L}_{n}(A / \mathrm{m})$ we say that $k^{\prime \prime} \in \operatorname{Obj} p-\mathrm{FGL}(A)$ is a lift of $F$ if $\bar{F}^{\prime}=F$; similarly for morphisms. The category of all such lifts over such an $A$ will be denoted by $\operatorname{lift}_{n}(A)$ and this is again a groupoid valued functor of $A$. Although lift $_{n}$ is not representable in Art $_{p}$, it is pro-representable in the sense of the following discussion.

Let $R$ be any graded commutative unital ring, and let $\mathbf{m}$ be a graded maximal ideal. Then we can define the $\mathrm{m}$-Artinian topology on $R$ to be the $R$-linear topology 
for which the open neighbourhoods of 0 are the ideals $J \triangleleft R$ with $J \subset \mathrm{m}$ and $R / J$ Artinian (we call such $J \mathbf{m}$-co-Artinian). Then the $\mathbf{m}$-Artinian completion of $R$ is $\hat{R} \lim R / J$. Similarly for any $R$-module we define

$$
\hat{M}=\lim _{J}(R / J \underset{R}{\otimes} M) \cong \lim _{J} M / J M
$$

In the case where $M$ is finitely generated, $\hat{M}$ is always linearly compact as in [4]. Observe that the ring $V(n)_{*}$ has $I=\left(v_{i}: i \neq n\right)$ as a graded maximal ideal. We will write $\widehat{V(n)_{*}}$ for the $I$-Artinian completion of $V(n)_{*}$ and define the Hopf algebroid $\widehat{(\widehat{V(n)}} *, \widehat{\left.\Gamma(n)_{*}\right)}$ by setting

$$
\widehat{\Gamma(n)}=\widehat{V(n)} \underset{V_{*}}{\otimes} \Gamma_{*} \underset{\nu_{*}}{\otimes} \widehat{V(n)}_{*}
$$

with the linear topology whose basic open ideals are generated by

$$
\eta_{R}\left(J_{1}\right)+\eta_{R}\left(J_{2}\right)
$$

for $J_{1}, J_{2} \in \widehat{V(n)}_{*}$ being co-Artinian in $\widehat{V(n)}_{*}$. From its definition the Artinian completion map $V(n)_{*} \rightarrow \widehat{V(n)_{*}}$ may be characterized as follows. Let $A \in \operatorname{Art}_{p}$ and let $f: V(n)_{*} \rightarrow A$ be continuous. Then there exists a unique continuous $\dot{\hat{f}: \widehat{V(n)}} \widehat{F}_{*} \rightarrow A$ making the diagram

commute. We then have

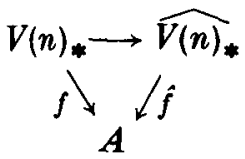

TheOREm 1.5. There are natural isomorphisms for $A \in \mathbf{A r t}_{p}$

$$
\left.\operatorname{lift}_{n}(A) \cong \operatorname{Hom}_{\text {cont }}\left(\widehat{\Gamma(n)}_{*}, A\right), \quad \operatorname{Obj~lift}_{n}(A) \cong \operatorname{Hom}_{\text {cont }}(\widehat{V(n)})_{*}, A\right) .
$$

We remark that $\widehat{V(n)}_{*}$ is an object of pro-Art ${ }_{p}$ and that

$$
\operatorname{Hom}_{\text {cont }}\left(\widehat{V(n)_{*}}, A\right) \cong \operatorname{pro-Art}_{p}\left(\widehat{V(n)}_{*}, A\right) ;
$$

however $\widehat{\Gamma(n)}_{*}$ is not in pro-Art ${ }_{p}$.

The pair $\left.\left(\widehat{V(n)_{*}}, \widehat{\Gamma(n)}\right)_{*}\right)$ inherits the structure of a topological Hopf algebroid.

A similar result holds for $\operatorname{lift}_{n}^{(n)}(A)=p$-FGL ${ }^{(n)}(A) \cap \operatorname{lift}_{n}(A)$ with $A \in \mathrm{Art}_{p}$. If $\widehat{E(n)}$ * is the Artinian completion of $E(n)_{*}$, then we can put

$$
\widehat{\Sigma(n)}_{*}=\widehat{E(n)_{*}} \underset{E(n)_{*}}{\otimes} \Sigma(n)_{*} \underset{E(n)_{*}}{\otimes} \widehat{E(n)_{*}}
$$

with the obvious linear topology. In fact $\widehat{E(n)}_{*}$ is noetherian, and we have

$$
\widehat{E(n)})_{*}=\underset{\leftarrow}{\lim } E(n)_{*} / I_{n}^{r}
$$

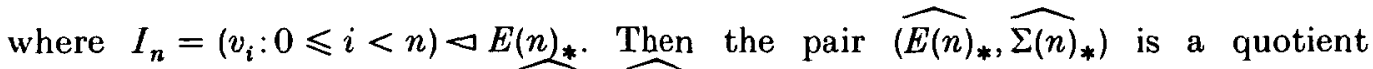
topological Hopf algebroid of $\left.(\widehat{V(n)}, \widehat{\Gamma(n)})_{*}\right)$ and we have 
Theorem 1.7. There are natural isomorphisms for $A \in \mathbf{A r t}_{p}$

$$
\begin{gathered}
\left.\operatorname{lift}_{n}^{(n)}(A) \cong \operatorname{Hom}_{\text {cont }} \widehat{\left(\Sigma(n)_{*}\right.}, A\right), \\
\operatorname{Obj} l i f t_{n}^{(n)}(A) \cong \operatorname{Hom}_{\text {cont }}\left(\widehat{E(n)_{*}}, A\right) \cong \operatorname{pro-Art}_{p}\left(\widehat{E(n)_{*}}, A\right) .
\end{gathered}
$$

Let $\phi \in \operatorname{lift}_{n}(A)$ with $A \in \mathbf{A r t}_{p}$. Then we say that $\phi$ is a ${ }^{*}$-isomorphism if

$$
\bar{\phi}(X)=X \in \mathbf{L}-\mathrm{T}_{n}(A / \mathbf{m}) \text {. }
$$

We denote by $\operatorname{lift}_{n}^{*}(A)$ the quotient groupoid

$$
\operatorname{lift}_{n}(A) /\left({ }^{*}\right. \text {-isomorphisms). }
$$

Our main result in this section is the following.

TheOREM 1.8. There is a idempotent natural equivalence of groupoid valued functors on $\mathbf{A r t}_{p}$

with im $\mathbf{e}(A)=\operatorname{lift}_{n}^{(n)}(A)$.

$$
\text { e: } \operatorname{lift}_{n}(A) \rightarrow \operatorname{lift}_{n}(A)
$$

Our main technical tool is the following infinite-dimensional version of Hensel's Lemma which may have other applications:

Theorem 1.9. Let $R$ be a local ring complete with respect to its maximal ideal $\mathbf{m}$. Let $\mathbf{X}=\left(X_{i}\right)_{i \geqslant 1}$ be an infinite sequence of indeterminates and $\mathbf{f}=\left(f_{j}\right)_{j \geqslant 1}$ be a sequence of polynomials $f_{j} \in R[\mathbf{X}]$ each of which only involves finitely many $X_{i}$. Suppose that $\mathbf{a}=\left(a_{i}\right)_{i \geqslant 1}$ is a sequence in $R$ for which

(a) $\mathbf{f}(\mathbf{a}) \equiv 0(\bmod \mathbf{m})$

(b) $M=d \mathbf{f}(\mathbf{a})$ satisfies over the field $\mathbf{k}=R / \mathbf{m}$ the condition

(*) for all $n$, the first $n$ rows of $M$ have $\mathbf{k}$-rank $n$.

Then there is a sequence $\mathbf{b}=\left(b_{i}\right)_{i \geqslant 1}$ in $R$ such that

$$
\mathbf{b} \equiv \mathbf{a}(\bmod \mathbf{m}) \quad \text { and } \quad \mathbf{f}(\mathbf{b})=0 .
$$

Proof. If $M$ is a doubly infinite matrix over a field $\mathbf{k}$, with each row finitely supported and satisfying $\left({ }^{*}\right)$, then by induction on $n$ we can row reduce the first $n$ rows into lower reduced echelon form with 1 as the last non-zero entry of each row and no other non-zero term below each such 1 . We can even assume that the induction is so arranged that any particular row eventually becomes constant.

Now putting $\mathbf{k}=R / \mathbf{m}$ we can perform such operations on $d \mathbf{f}(\mathbf{a})=M$ over $\mathbf{k}$. If we realize all of the row operations involved over $R$, then we have replaced $\mathbf{f}$ by an equivalent sequence of functions $\mathbf{g}$ with

$$
d \mathbf{g}(\mathbf{a})=E+N(\mathbf{a}) \equiv E(\bmod \mathbf{m})
$$

where $E$ is a matrix with 0 everywhere except for a single 1 occurring in each row in increasingly greater columns (we call such positions the pivots of $d g(a)$ ). Now if $N^{\prime}(a)$ is the result of removing from $N(\mathbf{a})$ each column with no pivot term, then

$$
N^{\prime}(a) \equiv \mathbf{0}(\bmod \mathbf{m})
$$


Now to solve for $\mathbf{b}$ we define a sequence (of sequences!) $\mathbf{b}^{(n)}$ by

$$
\begin{gathered}
\mathbf{b}^{(1)}=\mathbf{a}, \\
b_{i}^{(n)}=a_{i} \quad \text { if the } i \text { th column of } E \text { does not contain a pivot term, }
\end{gathered}
$$

and if $\mathbf{b}^{(n)}$ satisfies $\mathbf{g}\left(\mathbf{b}^{(n)}\right) \equiv \mathbf{0}\left(\mathbf{m}^{n}\right)$ then $\mathbf{b}^{(n+1)}$ is the unique solution $\left(\bmod \mathbf{m}^{n+1}\right)$ of

$$
d \mathbf{g}\left(\mathbf{b}^{(n)}\right)+d \mathbf{g}\left(\mathbf{b}^{(n)}\right)\left(\mathbf{b}^{(n+1)}-\mathbf{b}^{(n)}\right) \equiv \mathbf{0}\left(\bmod \mathbf{m}^{n+1}\right) .
$$

This solution is obtained on multiplying by

$$
\sum_{0 \leqslant r \leqslant n}\left(-N^{\prime}\left(\mathbf{b}^{(n)}\right)\right)^{r}
$$

and solving the resulting diagonal system for the remaining unknowns.

Clearly $\mathbf{b}^{(n)}$ is a Cauchy sequence and its limit solves the system

$$
\mathbf{f}(\mathbf{X})=\mathbf{0} \text {. }
$$

We will also need

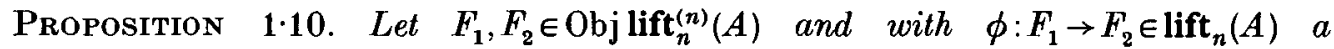
*-isomorphism. Then $F_{1}=F_{2}$ and $\phi(X)=X$.

Now we can proceed to prove Theorem 1-8. Let $F \in \mathrm{Obj} \mathrm{lift}_{n}(A)$, and $A \in \operatorname{Art}_{p}$. We will first construct a ${ }^{*}$-isomorphism $\mathrm{e}(F) \in \operatorname{lift}_{n}(A)$ with $\operatorname{dome}(F) \in \operatorname{lift}_{n}^{(n)}(A)$ and codom $\mathrm{e}(F)=F$. To do this it suffices to define a homomorphism

$$
\theta: \Gamma_{*} \rightarrow A
$$

extending the classifying homomorphism $\alpha: V_{*} \rightarrow A$ of $F$ and having $\theta\left(\eta_{R}\left(v_{n+\ell}\right)\right)=0$ for $i \geqslant 1$.

Now let

$$
f_{j}(\mathbf{t})=\eta_{R}\left(v_{n+j}\right) \in V_{*}\left[t_{i}: i \geqslant 1\right] .
$$

Then recall from $[13]$ that

$$
f_{j}(\mathbf{t}) \equiv v_{n} t_{j}^{p^{n}}-v_{n}^{p^{j}} t_{j}\left(\bmod \left(v_{k}: 0 \leqslant k \neq n\right)\right) .
$$

As $F \in \operatorname{Objlift}_{n}(A)$, we see that $\alpha\left(v_{n}\right)$ is a unit in $A$, and hence the polynomials $\alpha\left(f_{j}(\mathbf{t})\right) \in A\left[t_{i}: i \geqslant 1\right]$ satisfy the hypotheses of Theorem 1.9 with initial solution $(\bmod \mathbf{m})$ being $\mathbf{t}=\mathbf{0}$. We thus have a simultaneous solution for the $t_{f}$ and so can define a homomorphism $\theta$ as required.

Notice that by Proposition $1 \cdot 10$ there is only one such e $(F)$. In particular, if $F \in \operatorname{lift}_{n}^{(n)}(A)$ then $\mathbf{e}(F)=\operatorname{Id}_{F}$. To define $\mathrm{e}$ on morphisms we proceed as follows. Let $\phi: F_{1} \rightarrow F_{2} \in$ lift $_{n}(A)$. Then we define $\mathbf{e}(\phi)$ by requiring commutativity of the diagram

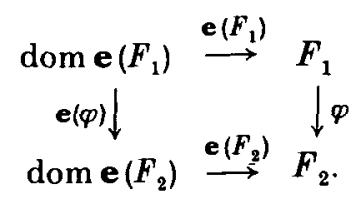

Clearly $(1 \cdot 10)$ also gives that $e$ is idempotent on morphisms.

Finally note that naturality is also a consequence of $(1 \cdot 10)$.

This proves Theorem $1 \cdot 8$. 


\section{Liftings of formal groups}

We now give the proof of Proposition 1/10. Let $\phi$ be such a strict isomorphism different from $X$. Then writing

we suppose that for all $i>0$,

$$
\phi(X)=\sum_{0 \leqslant i}^{F_{2}}\left(c_{i} X^{p^{i}}\right)
$$

and that for some $d>0$, we have

$$
c_{i} \equiv \mathbf{0}\left(\mathbf{m}^{r}\right)
$$

$$
c_{i} \begin{cases}\equiv 0\left(\mathbf{m}^{r+1}\right), & (0<i<d) \\ =0\left(\mathbf{m}^{r}\right), & (i=d) .\end{cases}
$$

Now we have

$$
[p]_{F_{1}} X=\sum_{0 \leqslant i \leqslant n}^{F_{1}}\left(u_{i} X^{p^{i}}\right), \quad[p]_{F_{2}} X=\sum_{0 \leqslant i \leqslant n}^{F_{2}}\left(v_{i} X^{p^{i}}\right)
$$

with $u_{i} \equiv v_{i} \equiv 0(\mathrm{~m})$ if $i<n$ and $u_{n}, v_{n}$ units. Now from formula (1-12) we can deduce that

$$
0 \equiv-u_{n}^{p^{d}} c_{d}\left(\mathbf{m}^{r+1}\right)
$$

(take $j=d)$. Hence $c_{d} \equiv 0\left(\mathbf{m}^{r+1}\right)$, contradicting our assumption.

The idempotent $\mathbf{e}$ is actually induced by morphisms of representing objects. Since we will need this for our applications and we are forced to work with prorepresentability, we will give details.

Now $\mathrm{e}$ is defined by assigning to each object $F$ in $\operatorname{lift}_{n}(A)$ a morphism $\mathrm{e}(F)$. From $(1 \cdot 5)$ we thus have a continuous ring homomorphism

$$
e(F): \widehat{\Gamma(n)}_{*} \rightarrow A
$$

classifying $\mathbf{e}(F)$. Moreover, precomposed with the left unit of $\widehat{\Gamma(n)}_{*}$ this agrees with the homomorphism

$$
\widehat{V(n)}_{*} \rightarrow A
$$

classifying $F$; on the other hand, precomposing with the right unit gives

$$
e_{0}(F): \widehat{V(n)}_{*} \rightarrow A
$$

which defines dom e $(F)$. Putting $A=V(n)_{*} / J$, an Artinian quotient of $V(n)_{*}$, and $F$ to equal the canonical group law, we obtain continuous homomorphisms

$$
e_{J}: \widehat{\Gamma(n)}_{*} \rightarrow V(n)_{*} / J, \quad\left(e_{J}\right)_{0}: \widehat{V(n)}_{*} \rightarrow V(n)_{*} / J
$$

By (1.9) we can lift these to unique continuous homomorphisms

$$
e: \widehat{\Gamma(n)}_{*} \rightarrow \widehat{V(n)}_{*}, \quad e_{0}: \widehat{V(n)}_{*} \rightarrow \widehat{V(n)}_{*}
$$

inducing e. By use of $(1 \cdot 10)$ we also can see that $e_{0}$ is idempotent. We have earlier identified ime with $\operatorname{lift}_{n}^{(n)}(A)$ and this is represented by the Hopf algebroid $\widehat{\left(E(n)_{*}\right.}, \widehat{\left.\Sigma(n)_{*}\right)}$. Again resorting to Artinian quotients of $V(n)_{*}$ we can construct a factorization

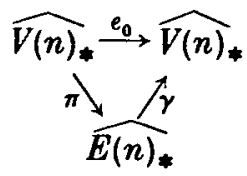


where $\pi$ is the canonical projection and $\gamma \pi$ is the identity. Hence $\gamma$ is an embedding. Now we can construct continuous morphisms of Hopf algebroids

$$
\begin{aligned}
& \tilde{\pi}:\left(\widehat{V(n)}_{*}, \widehat{\left.\Gamma(n)_{*}\right)} \rightarrow \widehat{\left(E(n)_{*}, \widehat{\Sigma(n)}\right.}\right) \text {, } \\
& \left.\left.\tilde{\gamma}: \widehat{(E(n)}, \widehat{\left.\Sigma(n)_{*}\right)} \rightarrow \widehat{\left(V(n)_{*}\right.}, \widehat{\Gamma(n)}\right)_{*}\right)
\end{aligned}
$$

which are the composites

$$
\begin{aligned}
& \pi \otimes 1 \otimes \pi: \widehat{V(n)}_{*} \otimes \Gamma_{*} \otimes \widehat{V(n)}_{*} \rightarrow \widehat{E(n)} * \Gamma \otimes \widehat{E(n)} \\
& \gamma \otimes 1 \otimes \gamma: \widehat{E(n)}_{*} \otimes \Gamma_{*} \otimes \widehat{E(n)}_{*} \rightarrow \widehat{V(n)_{*}} \otimes \Gamma \otimes \widehat{V(n)_{*}}
\end{aligned}
$$

(with all tensor products over $V_{*}$ ).

We can now extract the following key algebraic result.

Theorem 1·14. There is a unique *-isomorphism

$$
\mathrm{e}\left(F^{V}\right): e_{0 *}\left(F^{V}\right) \rightarrow F^{V}
$$

over $\widehat{V(n)}_{*}$ and the idempotent continuous ring homomorphism $e_{0}$ factors as

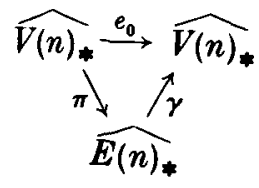

with $\pi$ the canonical projection and $\gamma$ injective.

More generally we can lift morphisms in $\mathbf{L}-\mathbf{T}_{n}(A / \mathbf{m})$.

Theorem 1·15. Let $\phi \in \mathbf{L}-\mathbf{T}_{n}(A / \mathbf{m})$. Then if $F \in \mathrm{Obj} \operatorname{lift}_{n}^{(n)}(A)$ with $\bar{F}=\operatorname{dom} \phi$ then there is a unique $\tilde{\phi} \in \operatorname{lift}_{n}^{(n)}(A)$ with

$$
\operatorname{dom} \tilde{\phi}=F \quad \text { and } \quad \overline{\tilde{\phi}}=\phi \in \mathrm{L}-\mathrm{T}_{n}(A / \mathrm{m})
$$

Proof. This uses (1.7) and (1.8).

Finally we remark that we have also shown

THEOREM 1·16. There is a natural equivalence of groupoids natural with respect to $A \in \mathbf{A r t}_{p}$

$$
\operatorname{lift}_{n}^{(n)}(A) \simeq \operatorname{lift}_{n}^{*}(A) .
$$

This is the precise form of the main result of [8] required in [10].

\section{The Artinian completions of $v_{n}^{-1} \mathrm{BP}$ and $E(n)$}

In this section we define the Artinian completions of certain spectra and explore some topological consequences of the results of Section 1.

Let $\mathbf{C W}^{f}$ be the category of finite CW-spectra. Let $h^{*}()$ be a cohomology theory on $\mathbf{C W}^{f}$ which is multiplicative with unit and commutative. Consider the functor on $\mathrm{CW}^{f}$

$$
\hat{h}^{*}()=\underset{J}{\lim }\left(h^{*} / J \bigotimes_{h^{*}} h^{*}()\right),
$$


where $J$ ranges over the Artinian open ideals of $h^{*}$ with respect to a given maximal ideal. Now in general, this functor is not a cohomology theory, since the functor

$$
M \rightarrow \underset{J}{\lim }\left(R / J \otimes_{R}^{\otimes} M\right)
$$

on $R$-modules is not always exact.

Recall that for a prime $p, \mathrm{BP}$ is a commutative ring spectrum; there are also associated ring spectra $v_{n}^{-1} \mathrm{BP}, E(n)$ (see [14]). Let $\mathbf{B P}_{0}$ denote the category of finitely generated $\mathrm{BP}_{*}(\mathrm{BP})$-comodules. Let $\mathrm{Mod}_{R}^{f}$ denote the category of finitely generated modules over a graded ring $R$. Then there are functors

$$
\begin{aligned}
& \alpha: \mathbf{B P}_{0} \rightarrow \operatorname{Mod}_{v_{n}^{-1} \mathbf{B P}_{*}}^{f} \\
& \beta: \mathbf{B P}_{0} \rightarrow \operatorname{Mod}_{E(n)_{*}}^{f}
\end{aligned}
$$

defined by $\quad \alpha(M)=v_{n}^{-1} M \cong v_{n}^{-1} B P_{*} \otimes M \quad$ and $\quad \beta(M)=E(n)_{*} \otimes M$,

where the tensor products are over $\mathrm{BP}_{*}$. Now the Artinian completion functor of Section 1 defines

$$
\begin{aligned}
& \wedge: \operatorname{Mod}_{v_{n}^{-1} \mathrm{BP}_{*}}^{f} \rightarrow \operatorname{Mod}_{v_{n}^{-1} \mathrm{BP}}^{\mathrm{lc}} \\
& \wedge: \operatorname{Mod}_{E(n)_{*}}^{f} \rightarrow \operatorname{Mod}_{\overparen{E(n)}}^{\mathrm{lc}}
\end{aligned}
$$

(where lc denotes linearly compact). We have

TheOREM 2:1. The composite functors

$$
\begin{aligned}
\operatorname{BP}_{0} \stackrel{\alpha}{\rightarrow} \operatorname{Mod}_{v_{n}^{-1} B P_{*}}^{f} \stackrel{\wedge}{\rightarrow} \operatorname{Mod}_{v_{n}^{-1} \mathrm{BP}_{*}}^{\mathrm{lc}} & \stackrel{\wedge}{\rightarrow} \operatorname{Mod}_{\overparen{E(n)}}^{\mathrm{lc}}
\end{aligned}
$$

are exact. Moreover there are natural isomorphisms (of $\widehat{v_{n}^{-1} \mathrm{BP}_{*^{-}}}$and $\widehat{E(n)}_{*^{-m o d u l e s}}$ )

$$
\begin{gathered}
{\widehat{v_{n}^{-1} \mathrm{BP}}}_{*} \otimes M \cong \alpha(M), \\
\widehat{E(n)}_{*} \otimes M \cong \beta(M),
\end{gathered}
$$

on the category $\mathbf{B P}_{0}$. In particular it follows that $\widehat{v_{n}^{-1} \mathrm{BP}^{*}() \text { and } \widehat{E(n)}} \widehat{E}^{*}()$ are cohomology theories on $\mathrm{CW}^{f}$.

Before proving this we will explore some consequences. We will rely heavily on the following algebraic fact.

Leмма 22. Let $R$ be a unital ring. Let $\left\{M_{\alpha}\right\}$ be an inverse system of topological $R$ modules which are linearly compact. Then

$$
\underset{\alpha^{-}}{\lim _{\alpha}^{(n)}} M_{\alpha}=0 \text { if } n>0
$$

Proof. See $[4,18]$.

We have the following results, all consequences of $(2 \cdot 2)$.

Proposition 2-3. Suppose $\hat{h}^{*}()$ is a cohomology theory on $\mathbf{C W}^{f}$ and that $k^{*}()$ is 
another cohomology theory on $\mathbf{C W}^{f}$. Let $K$ and $\hat{H}$ be spectra representing these theories. If $\phi: k^{*}() \rightarrow \hat{h}^{*}()$ is a natural transformation, then there is a unique morphism of spectra $\hat{\Phi}: K \rightarrow \hat{H}$ inducing $\phi$.

In particular, if we take $k^{*}()=\hat{h}^{*}()$ then we see that $\hat{H}$ is unique up to equivalence. Thus $\hat{h}^{*}()$ admits a unique representable extension to the stable homotopy category $\mathbf{C W}$.

Proof. Let $K=\underset{\alpha}{\operatorname{holim}} K_{\alpha}$ with $K_{\alpha} \in \mathrm{CW}^{f}$. Then we have a spectral sequence of [1], $\frac{\lim }{\alpha}^{(s)} \hat{h}^{*-8}\left(K_{\alpha}\right) \Rightarrow \hat{h}^{*}(K)$ which by $(2 \cdot 1)$ gives

$$
\hat{h}^{*}(K) \cong \lim _{\alpha} h^{*}\left(K_{\alpha}\right)
$$

Yoneda's Lemma now completes the proof.

Proposition 2.4. $\hat{H}$ has a unique ring spectrum structure compatible with the canonical natural transformation being multiplicative.

$$
h^{*}() \rightarrow \hat{h}^{*}()
$$

Proof. Let $\hat{H}=\underset{\alpha}{\operatorname{holim}} X_{\alpha}$, with $X_{\alpha} \in \mathbf{C W}^{f}$. Then there is a pairing

$$
\hat{h}^{*}\left(X_{\alpha}\right) \otimes \hat{h}^{*}\left(X_{\beta}\right) \rightarrow \hat{h}^{*}\left(X_{\alpha} \wedge X_{\beta}\right)
$$

and hence as

$$
\hat{H} \wedge \hat{H} \simeq \underset{(\alpha, \beta)}{\operatorname{holim}} X_{\alpha} \wedge X_{\beta}
$$

Adams' spectral sequence again gives (using $(2 \cdot 1)$ )

$$
\hat{h}^{*}(\hat{H} \wedge \hat{H}) \cong \lim _{(\alpha, \beta)} \hat{h}^{*}\left(X_{\alpha} \wedge X_{\beta}\right)
$$

Once more Yoneda's Lemma shows that there is a unique map $\hat{H} \wedge \hat{H} \rightarrow \hat{H}$ compatible with the product on $h^{*}()$. Associativity and commutativity also follow in this fashion.

Proof of $2 \cdot 1$. Let $g_{i} \in v_{n}^{-1} \mathrm{BP}{ }_{*}$, with $i \geqslant 1$, be a sequence of elements of form

$$
g_{i}=v_{n+i}-f_{i}
$$

where $f_{i} \in \mathbb{Z}_{(p)}\left[v_{1}, \ldots, v_{n}, v_{n}^{-1}, v_{n+1}, \ldots, v_{n+i-1}\right] \in v_{n}^{-1} \mathrm{BP}{ }_{*}$. We can then define a sequence of ideals $J_{i} \triangleleft v_{n}^{-1} \mathrm{BP}_{*}$ with $i \geqslant 1$ and

$$
J_{i}=\left(g_{j}: j \geqslant i\right) \text {. }
$$

Now for each $i, v_{n}^{-1} \mathrm{BP}_{*} / J_{i}$ satisfies the hypotheses of Landweber's exact functor theorem [7] and so the functor

$$
M \rightarrow v_{n}^{-1} \mathrm{BP}_{*} / J_{i} \otimes M
$$

is exact on $\mathbf{B P} \mathbf{P}_{0}$. Notice that $v_{n}^{-1} \mathrm{BP} \mathbf{P}_{*} / J_{i}$ is Noetherian. There is a maximal ideal

$$
\mathbf{m}(i)=\left(v_{l}: 0 \leqslant l<n+i, l \neq n\right) \subset v_{n}^{-1} \mathrm{BP}_{*} / J_{i}
$$


and each quotient $\left(v_{n}^{-1} \mathrm{BP}_{*} / J_{i}\right) / \mathbf{m}(i)^{r}$ is Artinian and local. Moreover, we have by standard facts on completions (see [9]), that if $M$ is finitely generated over $v_{n}^{-1} \mathrm{BP} * / J_{i}$ then

$$
\left.\underset{r}{[\lim }\left(v_{n}^{-1} \mathrm{BP}{ }_{*} / J_{i}\right) / \mathbf{m}(i)^{r}\right] \otimes M \cong \underset{r}{\lim } M / \mathbf{m}(i)^{r} M
$$

and $\underset{r}{\lim }\left(v_{n}^{-1} \mathrm{BP}_{*} / J_{i}\right) / \mathrm{m}(i)^{r}$ is flat over $v_{n}^{-1} \mathrm{BP}_{*} / J_{i}$. Because $M / \mathrm{m}(i)^{r} M$ is Artinian, from [4] we know that this inverse limit is linearly compact.

We thus see that for any finitely generated module $M$ over $v_{n}^{-1} \mathrm{BP}_{*}$, the functor

is exact by $(2 \cdot 2)$.

$$
\lim _{i} \underset{r}{\lim }\left[\left(v_{n}^{-1} \mathrm{BP}_{*} / J_{i}\right) / \mathbf{m}(i)^{r} \otimes M\right]
$$

Finally, observe that if $J$ is a co-Artinian ideal of $v_{n}^{-1} \mathrm{BP}_{*}$ with respect to the standard maximal ideal $\mathrm{m}=\left(v_{i}: 0 \leqslant i \neq n\right)$ then it must contain an ideal of form $J_{i}=\left(g_{j}: j \geqslant i\right)$ for some sequence $g_{i}$ as above, since $v_{n}^{-1} \mathrm{BP} * / J$ is also Noetherian (see [9]). Hence we see that the ideals

$$
\mathbf{m}(i)^{r}+J_{i}
$$

are in fact cofinal in the Artinian open sets of $v_{n}^{-1} \mathrm{BP}_{*}$ with respect to the maximal ideal $\mathbf{m}$. This shows that the functor $M \rightarrow \widehat{v_{n}^{-1} M}$ is exact on $\mathbf{B P}_{0}$. The argument in the $E(n)$ case is direct since $E(n)_{*}$ is Noetherian. Because $\mathrm{BP}_{*}$ is a coherent ring, $M$ admits a finite presentation $0 \rightarrow N \rightarrow F \rightarrow M \rightarrow 0$ with $F$ finitely generated and free over $\mathrm{BP}_{*}$. Consider the induced commutative diagram with exact rows :

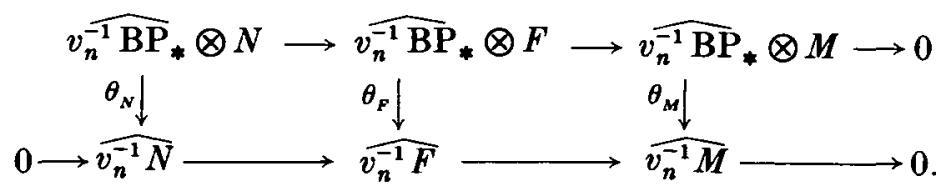

Now $\theta_{F}$ is clearly an isomorphism and hence $\theta_{M}$ is surjective. Replacing $M$ by $N$ and repeating this argument shows that $\theta_{N}$ is surjective. Finally a diagram chase shows that $\theta_{M}$ is also injective.

This discussion proves Theorem $2 \cdot 1$.

We now derive our main results on splitting $\widehat{v_{n}^{-1} \mathrm{BP}}$ as an $\widehat{E(n)}$-module spectrum. Let $x^{\mathrm{BP}} \in \mathrm{BP}^{2}\left(\mathbb{C} P^{\infty}\right)$ be the canonical $\mathbb{C}$-orientation of [1]. The canonical maps of ring spectra

$$
\begin{gathered}
\mathrm{BP} \rightarrow v_{n}^{-1} \mathrm{BP} \rightarrow \widehat{v_{n}^{-1} \mathrm{BP}} \\
\mathrm{BP} \rightarrow E(n) \rightarrow \widehat{E(n)}
\end{gathered}
$$

induce $\mathbb{C}$-orientations on $\widehat{v_{n}^{-1} \mathrm{BP}}$ and $\widehat{E(n)}$, and under the identifications

$$
\widehat{V(n)}_{*} \cong{\widehat{v_{n}^{-1} \mathrm{BP}}}_{*} \cong \pi_{*} \widehat{\left(v_{n}^{-1} \mathrm{BP}\right)}, \quad \widehat{E(n)}{ }_{*} \cong \pi_{*} \widehat{(E(n))}
$$

the orientations $x^{V}, x^{E}$ have associated group laws $F^{V}, F^{E}$. We have shown in (1.14) that there is a *-isomorphism

$$
\mathrm{e}\left(F^{V}\right):\left(e_{0}\right) *\left(F^{V}\right) \rightarrow F^{V}
$$


and that this is induced by an idempotent continuous ring homomorphism

$$
e_{0}:{\widehat{v_{n}^{-1} \mathrm{BP}_{*}}} \widehat{E(n)}_{*} \rightarrow{\widehat{v_{n}^{-1} \mathrm{BP}}}_{*} \text {. }
$$

Theorem 25. There is a unique idempotent multiplicative natural transformation

$$
\bar{E}_{n}: \widehat{v_{n}^{-1 \mathrm{BP}}} *() \rightarrow \widehat{v_{n}^{-1} \mathrm{BP}} *()
$$

such that on $\mathbb{C} P^{\infty}$ we have

$$
\bar{E}_{n}\left(x^{V}\right)=\mathbf{e}\left(F^{V}\right)^{-1}\left(x^{V}\right) .
$$

Moreover, we have a canonical natural isomorphism

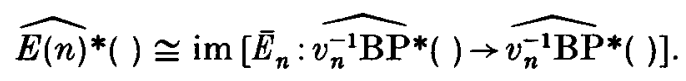

Using our strong representability results for these functors, we deduce the following conjecture of D. Ravenel [14].

THEOREM 2·6. There is a splitting of $\widehat{E(n)}$-module spectra

$$
\widehat{v_{n}^{-1} \mathrm{BP}} \simeq \prod_{\alpha} \Sigma^{\sigma(\alpha)} \widehat{E(n)}
$$

and the natural morphism of ring spectra $\widehat{v_{n}^{-1} \mathrm{BP}} \rightarrow \widehat{E(n)}$ splits as a morphism of $\widehat{E(n)}$. module spectra.

Proof of (2.5). By the universal property of BP, we have a natural transformation of multiplicative theories

$$
\bar{E}_{n}^{\prime}: \mathrm{BP}^{*}() \rightarrow \widehat{v_{n}^{-1} \mathrm{BP}^{*}()}
$$

sending $x^{\mathrm{BP}}$ to $\mathrm{e}^{*}\left(F^{V}\right)^{-1}\left(x^{V}\right)$. Now for $Z \in \mathbf{C W}^{f}$,

$$
{\widehat{v_{n}^{-1} \mathrm{BP}}}^{*}(Z) \cong \widehat{v_{n}^{-1} \mathrm{BP}} * \otimes \mathrm{BP}^{*}(Z)
$$

by $(2 \cdot 1)$, and hence we can extend $\bar{E}_{n}^{\prime}$ to a natural transformation

$$
\bar{E}_{n}:{\widehat{v_{n}^{-1} \mathrm{BP}}}_{*} \otimes \mathrm{BP}^{*}() \rightarrow \widehat{v_{n}^{-1} \mathrm{BP}^{*}} *()
$$

on $\mathbf{C W}^{f}$ by applying $e_{0}$ on the left-hand factor of the tensor product, which is of course isomorphic to $\widehat{v_{n}^{-1} \mathrm{BP}} *()$. But now by $(2 \cdot 3,4)$ we have a unique multiplicative natural transformation $\bar{E}_{n}$ induced by a unique morphism of ring spectra

$$
E_{n}: \widehat{v_{n}^{-1} \mathrm{BP}} \rightarrow \widehat{v_{n}^{-1} \mathrm{BP}} .
$$

On coefficient rings this agrees with $e_{0}$. Notice that $\bar{E}_{n}^{2}=\bar{E}_{n}$ since

$$
\bar{E}_{n} \bar{E}_{n}^{\prime}=\bar{E}_{n}^{\prime}
$$

by the fact that $\mathrm{e}$ is idempotent. By $(2 \cdot 3)$ we see that $E_{n}^{2}=E_{n}$. Finally, the theory $\operatorname{im} \bar{E}_{n}$ has a natural transformation

$$
\operatorname{im} \bar{E}_{n} \rightarrow \widehat{v_{n}^{-1} \mathrm{BP}} *() \rightarrow \widehat{E}(\widehat{E}(n) *()
$$


and from $(2 \cdot 3)$ and Whitehead's Theorem we deduce that this is a natural isomorphism. Hence we obtain a morphism of ring spectra

$$
\rho: \widehat{E(n)} \rightarrow \widehat{v_{n}^{-1} \mathrm{BP}}
$$

inducing this inclusion of theories.

Of course, $\rho$ makes $\widehat{v_{n}^{-1} \mathrm{BP}}$ a module spectrum over $\widehat{E(n)}$.

Proof of $2 \cdot 6$. We need only show that as $\widehat{E(n)})_{*}$-module theories

$$
\widehat{v_{n}^{-1} \mathrm{BP}^{*}}() \cong \prod_{\alpha} \widehat{E(n)^{*+\sigma(\alpha)}}() \cong\left[\quad, \prod_{\alpha} \Sigma^{\sigma(\alpha)} \widehat{E(n)}\right] \text {. }
$$

Let $Z \in \mathbf{C W}^{f}$. Then we will construct a (natural in $Z$ ) continuous morphism of $\widehat{E(n)}_{*^{-}}$modules

for suitable indexing set $\{\alpha\}^{\alpha}$

$$
\Pi \widehat{E(n)}^{*+\sigma(\alpha)}(Z) \rightarrow{\widehat{v_{n}^{-1} \mathrm{BP}^{*}}}^{*}(Z)
$$

As an $\widehat{E(n)}_{*}$-module (via $\rho_{*}$ ) $\widehat{v}_{n}^{-1 \mathrm{BP}}{ }_{*}$ is free topological on the set of monomials $v_{n+1}^{r_{1}} \ldots v_{n+k}^{r_{k}}$, with each $r_{i} \geqslant 0$. Observe that almost all of these are in a given coArtinian ideal $J \triangleleft v_{n}^{-1} \mathrm{BP}_{*}$, hence for any element $\left\{x_{v}\right\} \in \Pi_{v} \widehat{E(n)}{ }^{*+|v|}(Z)$ we find that in $v_{n}^{-1} \mathrm{BP} *(Z) / J$ the sum $\Sigma_{v} v x_{v}$ reduces to a finite sum. Here $v$ ranges over the above monomials. From these quotients we can obtain a lift to $\widehat{v_{n}^{-1} \mathrm{BP}} *(Z)$ which corresponds to the above series. It is easy to verify this given an isomorphism of theories by taking $Z=S^{0}$. Then Whitehead's Theorem and $(2 \cdot 3)$ give the result.

We remark that there are other situations in which our methods apply. Let $P(m)$ denote the spectrum of [14] with $P(m)_{*}=\mathbb{F}_{p}\left[v_{m+i}: i \geqslant 0\right]$ and $E(m, n)$ (for $\left.m \leqslant n\right)$ the spectrum obtained by Baas-Sullivan theory with $E(m, n)_{*}=\mathbb{F}_{p}\left[v_{m}, v_{m+1}, \ldots, v_{n}, v_{n}^{-1}\right]$. We can form Artinian completions of these and then we have a splitting of $E(m, n)$ module spectra

$$
\widehat{v_{n}^{-1} P(m)} \simeq \prod_{\gamma} \Sigma^{\sigma(\gamma)} \widehat{E(m, n)} .
$$

For $m=n$ our methods agree with those of [17], which also contains a proof of the necessity of some form of completion to obtain such module spectra splittings.

Notice also that the case $n=1$ of $(2 \cdot 6)$ shows that the $p$-complete version of Adams' summand of $K U_{(p)}$ completely determines $\widehat{v_{1}^{-1} \mathrm{BP}}$, which is a sort of converse to the classical Conner-Floyd Theorem.

\section{Some change of ring isomorphisms}

In this section we describe some homological consequences of our earlier work. In particular, we reprove some 'change of ring' isomorphisms for certain Ext groups which play a central part in computations of the Adams-Novikov $E_{2}$-term. We refer the reader to [13], especially chapter 6 , for basic definitions and results.

Let $\left(A_{1}, \Gamma_{1}\right)$ and $\left(A_{2}, \Gamma_{2}\right)$ be Hopf algebroids, and $f, g:\left(A_{1}, \Gamma_{1}\right) \rightarrow\left(A_{2}, \Gamma_{2}\right)$ be two morphisms. Then a ring homomorphism $H: \Gamma_{1} \rightarrow A_{2}$ is a natural equivalence from $f$ to $g$ if

$$
H \eta_{L}=\epsilon f \eta_{L}: A_{1} \rightarrow A_{2} \text { and } H \eta_{R}=\epsilon g \eta_{R}: A_{1} \rightarrow A_{2} .
$$

Given any left $\Gamma_{1}$-comodule $N$ we can define a $\Gamma_{2}$-comodule $f^{*} N$ by

$$
f * N=A_{2} \underset{A_{1}}{\otimes} N
$$


with coproduct formed from the composition

$$
A_{2} \underset{A_{1}}{\otimes} \underset{1 \otimes \psi_{N}}{\longrightarrow} A_{2} \underset{A_{1}}{\otimes} \Gamma_{1} \underset{A_{1}}{\otimes} \underset{1 \otimes f \otimes 1}{\longrightarrow} A_{2} \underset{A_{2}}{\otimes} \Gamma_{2} \underset{A_{2}}{\otimes} A_{2} \underset{A_{1}}{\otimes} \underset{\cong}{\longrightarrow} \underset{C_{2}}{\otimes} \underset{A_{2}}{A_{2}} \underset{A_{1}}{\otimes} N
$$

There is then an induced homomorphism

$$
f_{*}: \operatorname{Ext}_{\Gamma_{1}}^{*}\left(A_{1}, N\right) \rightarrow \operatorname{Ext}_{\Gamma_{2}}^{*}\left(A_{2}, A_{2} \underset{A_{1}}{\otimes} N\right)
$$

and we have (by a slight strengthening of [13]),

Proposition 3·1. If $f, g:\left(A_{1}, \Gamma_{1}\right) \rightarrow\left(A_{2}, \Gamma_{2}\right)$ are naturally equivalent then

$$
f_{*}=g_{*}: \operatorname{Ext}_{\Gamma_{1}}^{*}\left(A_{1}, N\right) \rightarrow \operatorname{Ext}_{\Gamma_{2}}^{*}\left(\Gamma_{2}, A_{2} \underset{A_{1}}{\otimes}\right) .
$$

Proof. A natural equivalence $H$ induces a chain homotopy (in the cobar complex) between the maps induced by $f$ and $g$.

If $f:\left(A_{1}, \Gamma_{1}\right) \rightarrow\left(A_{2}, \Gamma_{2}\right)$ and $h:\left(A_{2}, \Gamma_{2}\right) \rightarrow\left(A_{1}, \Gamma_{1}\right)$ are morphisms such that $h f$ is naturally equivalent to the identity of $\left(A_{1}, \Gamma_{1}\right)$ and $f h$ to that of $\left(A_{2}, \Gamma_{2}\right)$, then we say that $f$ and $h$ are inverse equivalences of $\left(A_{1}, \Gamma_{1}\right)$ and $\left(A_{2}, \Gamma_{2}\right)$. Then we have

Proposition 3·2. Let $f, h$ be inverse equivalences of $\left(A_{1}, \Gamma_{1}\right)$ and $\left(A_{2}, \Gamma_{2}\right)$. Then for any $\Gamma_{1}$-comodule $N$ there are natural isomorphisms

and

$$
\begin{gathered}
\operatorname{Ext}_{\Gamma_{1}}^{*}\left(A_{1}, N\right) \cong \operatorname{Ext}_{\Gamma_{2}}^{*}\left(A_{2}, f^{*} N\right) \\
\operatorname{Ext}_{\Gamma_{1}}^{*}\left(A_{1}, N\right) \cong \operatorname{Ext}_{\Gamma_{1}}^{*}\left(A_{1}, h^{*} f^{*} N\right) .
\end{gathered}
$$

This result follows easily from $(3 \cdot 1)$.

We can now apply these results to the Hopf algebroids of Section 1. Notice that the morphisms of topological Hopf algebroids

$$
\begin{aligned}
& \tilde{\pi}:\left(\widehat{V(n)_{*}}, \widehat{\Gamma(n)_{*}}\right) \rightarrow\left(\widehat{E(n)}, \widehat{\Sigma(n)_{*}}\right) \\
& \left.\tilde{\gamma}: \widehat{(\widehat{E(n)}}, \widehat{\Sigma(n)_{*}}\right) \rightarrow\left(\widehat{V(n)_{*}}, \widehat{\Gamma(n)_{*}}\right)
\end{aligned}
$$

are inverse equivalences since $\tilde{\pi} \tilde{\gamma}$ is the identity and $\tilde{\gamma} \tilde{\pi}$ is naturally equivalent to the identity using the homomorphism $e: \widehat{\Gamma(n)}_{*} \rightarrow \widehat{V(n)_{*}}$. Hence we have

Theorem 3-3. For any $\widehat{\Gamma(n)}_{*}$-comodule $N$, there is a natural isomorphism

$$
\left.\operatorname{Ext}_{\widehat{\Gamma(n)}}^{*}\left(\widehat{V(n)_{*}} N\right) \cong \operatorname{Ext}_{\overparen{\Sigma(n)} *}^{*} \widehat{\left(E(n)_{*}\right.}, \widehat{E(n)_{*}} \underset{\widehat{V(n)}}{\otimes} N\right)
$$

Corollary 344. Let $N$ be a $\mathrm{BP}{ }_{*} \mathrm{BP}$-comodule in which every element is $I_{n}$-torsion and $v_{n}$ acts bijectively. Then there is a natural isomorphism

$$
\operatorname{Ext}_{\mathrm{BP}_{*} \mathrm{BP}^{*}}\left(\mathrm{BP}_{*}, N\right) \cong \operatorname{Ext}_{\Sigma(n)_{*}}^{*}\left(E(n)_{*}, E(n)_{*} \underset{\mathrm{BP}_{*}}{\otimes} N\right)
$$

Proof. Using the cobar complex, we can easily verify that

$$
\operatorname{Ext}_{\mathrm{BP}, \mathrm{BP}}^{*}\left(\mathrm{BP}_{*}, N\right) \cong \operatorname{Ext}_{\Gamma(n) *}^{*}\left(V(n)_{*}, V(n)_{*} \underset{\mathrm{BP}_{*}}{\otimes} N\right)
$$


Exactly as in the proof of $[11]$, theorem $3 \cdot 10$ we can reduce to the case in which $N=V(n)_{*} / I_{n}$. Hence we must consider the commutative diagram

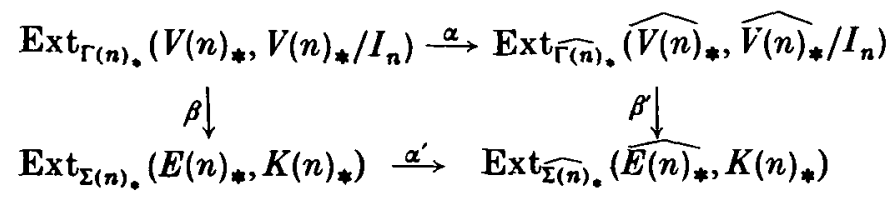

where $\alpha, \alpha^{\prime}, \beta, \beta^{\prime}$ are the canonical maps. Using [11], proposition $1 \cdot 3$ we see that $\alpha^{\prime}$ is an isomorphism since its domain and codomain are isomorphic to

$$
\operatorname{Ext}_{K(n), K(n)}\left(K(n)_{*}, K(n)_{*}\right)
$$

By our Theorem $3 \cdot 3, \beta^{\prime}$ is an isomorphism, hence $(3 \cdot 4)$ follows if we can show that $\alpha$ is an isomorphism.

Let $\widetilde{\Gamma(n)}_{*}=\Gamma(n)_{*} \otimes_{V(n)} \widehat{V(n)_{*}}$ considered as a right $\widehat{\Gamma(n)_{*}}$-comodule in the obvious fashion. It is not hard to show that

and

$$
\begin{gathered}
{\widetilde{\Gamma(n)_{*}}}^{\square} \widehat{\Gamma(n)}_{*} \widehat{V(n)}_{*} \cong V(n)_{*} \\
{\widetilde{\Gamma(n)_{*}}}_{\widetilde{\Gamma(n)}_{*}} \widehat{V(n)}_{*} / I_{n} \cong V(n)_{*} / I_{n} .
\end{gathered}
$$

To prove $\alpha$ is surjective we will interpret elements of Ext $\mathrm{t}^{s}$ as long exact sequences. Let $[E] \in \operatorname{Ext}_{\widehat{\Gamma(n)}}^{s}\left(\widehat{V(n)_{*}}, \widehat{V(n)_{*}} / I_{n}\right)$, where $s>0$, be the class of the exact sequence

$$
E: 0 \rightarrow \widehat{V(n)}_{*} / I_{n} \rightarrow M_{1} \rightarrow \ldots \rightarrow M_{s} \rightarrow \widehat{V(n)}_{*} \rightarrow 0
$$

of $\widehat{\Gamma(n)}{ }_{*}$-comodules. Then the sequence

$$
F=\widetilde{\Gamma(n)}_{*} \square \widehat{\Gamma(n)} E
$$

is a sequence of left $\Gamma(n)_{*}$-comodules. From Section 2 we know that $\widehat{V(n)}_{*} \otimes_{V(n)}$ is exact on finitely generated $\Gamma(n)_{*}$-comodules, and it is in fact faithfully flat on this category (see [9]). Now

$$
\widehat{V(n)}_{*} \underset{V(n) *}{\otimes} F \cong E
$$

and by faithful flatness, $F$ is also exact. Hence by $(3 \cdot 5,6)$

$$
[f] \in \operatorname{Ext}_{\Gamma(n)_{*}}^{s}\left(V(n)_{*}, V(n)_{*} / I_{n}\right)
$$

and

$$
\alpha([F])=[E] .
$$

The case $s=0$ is proved by noting that

$$
\operatorname{Ext}_{K(n)_{*} K(n)}^{0}\left(K(n)_{*}, K(n)_{*}\right)=K(n)_{*}
$$

and that

$$
v_{n}^{k} \in \mathrm{Ext}_{\Gamma(n)}^{0}\left(V(n)_{*}, V(n)_{*} / I_{n}\right)
$$

maps onto $v_{n}^{k} \in K(n)_{*}$ - hence $\alpha$ is surjective for $s \geqslant 0$.

To prove injectivity, we consider for $s \geqslant 2$ an exact sequence of $\Gamma(n)_{*}$-comodules

$$
F: 0 \rightarrow V(n)_{*} / I_{n} \rightarrow N_{1} \rightarrow \ldots \rightarrow N_{s} \rightarrow V(n)_{*} \rightarrow 0
$$


and suppose $\alpha([F])=0$. Then there are $s$-fold extensions $E_{i}$ for $1 \leqslant i \leqslant 2 k$ with

$$
\widehat{V(n)}_{*} \otimes F \rightarrow E_{1} \leftarrow E_{2} \rightarrow \ldots \leftarrow E_{2 k}=0
$$

where 0 is the trivial extension. Applying $\widetilde{\Gamma(n)} * \square \widehat{\Gamma(n)}$, and using $(3 \cdot 5,6)$ together with faithful flatness of $V(n)_{*}$ gives $[F]=0$. The case $s=0$ is clear since the map $V(n)_{*} / I_{n} \rightarrow \widehat{V(n)_{*}} / I_{n}$ is monic. The case $s=1$ follows by a modification of the argument for $s \geqslant 2$. Hence we have shown that $\alpha$ is an isomorphism and so proved $(3 \cdot 4)$.

Corollary 3·7. The natural projection $\mathrm{BP}_{*} \rightarrow K(n)_{*}$ induces an isomorphism

$$
\operatorname{Ext}_{\mathrm{BP}}^{*} \mathrm{BP}\left(\mathrm{BP}_{*}, v_{n}^{-1} \mathrm{BP}_{*} / I_{n}\right) \cong \mathrm{Ext}_{K(n)_{*} K(n)}^{*}\left(K(n)_{*}, K(n)_{*}\right) \text {. }
$$

Proof. This follows immediately from $(3 \cdot 4)$ and [11], proposition 1.3 since

$$
K(n)_{*} \cong E(n)_{*} / I_{n} \text {. । }
$$

Of course Corollaries 3.4 and 3.7 form the main results of [11].

We remark also that similar results are possible for topological comodules. However, except for well behaved topologies, there are technical difficulties in dealing with these, so we refrain from making such statements here.

\section{4. $K(n)$ localization and Artinian completion}

In this section we consider our homotopy theoretic results in the light of Ravenel's work [14] and recent results of Devinatz, Hopkins and Smith (see especially [3]). We will prove

TheOREM 4:1. The natural map

$$
E(n) \rightarrow \widehat{E(n)}
$$

is a $K(n)$ localization and $\widehat{v_{n}^{-1} \mathrm{BP}}$ is $K(n)$ local.

We begin by recalling some results of [3]. Let $X$ be a finite CW-spectrum. Then for a prime $p$ and $n>0$ we say that $X$ has class $\mathbf{C}_{n}$ (written $X \in \mathbf{C}_{n}$ ) if $X$ is $p$-local and is $E(n-1)$ acyclic. By [14] this last requirement is equivalent to $X$ being $\bigvee_{0 \leqslant j<n} K(j)$ acyclic. If $X \in C_{n}$ we say that a (graded) morphism of spectra $\theta: X \rightarrow X$ is a $v_{n}$-self map if $\theta_{*}: K(m)_{*}(X) \rightarrow K(m)_{*}(X)$ is an isomorphism if $m=n$, and is nilpotent if $m \neq n$. A major result of [3] is

Theorem 4.2. (a) For $X \in \mathrm{C}_{n}$ there is a $v_{n}$-self map

$$
\theta_{X}: X \rightarrow X
$$

(b) For $X, Y \in \mathbf{C}_{n}$ and a map $f: X \rightarrow Y$ there are positive integers $r, s$ such that

commutes.

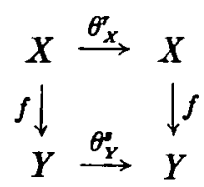


Now let $X$ be an arbitrary spectrum and $C_{n}(X)$ the following category. The objects are pairs $(Y, f)$ where $Y \in \mathrm{C}_{n}$ and $f: X \rightarrow Y$ is a morphism of spectra. The morphisms $\phi:(Y, f) \rightarrow(Z, g)$ are morphisms of spectra $\phi: Y \rightarrow Z$ such that

commutes.

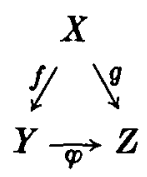

Observe that for $\phi, \psi:(Y, f) \rightarrow(Z, g)$ there is an equalizer (Cone $(\phi-\psi), h g$ ) where $h: Z \rightarrow$ Cone $(\phi-\psi)$ is the canonical map. Also, if $(Y, f),(Z, g)$ are objects, then there exists an object $(Y \vee Z, k)$ and there exist morphisms

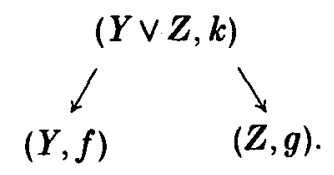

This last fact shows that $C_{n}(X)$ is filtered. We can thus define inverse limits indexed on $\mathbf{C}_{n}(X)$, at least if we accept that $\mathbf{C}_{n}(X)$ is small. For $X$ finite this is so, since there is a cofinal small subcategory $C_{n}^{\prime}(X)$ to be described in the following discussion. We will set

if any problem arises here.

$$
\underset{\mathrm{c}_{n}(x)}{\lim }=\underset{\mathrm{c}_{n}^{\prime}(X)}{\lim }
$$

Now let $X$ be a finite CW-spectrum. Then for an object $(Y, f)$ in $\mathrm{C}_{n}(X)$ consider the following inductive construction.

As $Y$ is finite, for some $r$ the self map $p^{r}$ on $Y$ is trivial; hence we have a diagram

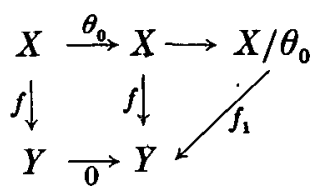

where $\theta_{0}=p^{r}$ on $X$ is a $K(0)$ equivalence and the row is a cofibre sequence.

Now suppose that for $1 \leqslant k \leqslant n-1$ we have constructed a diagram

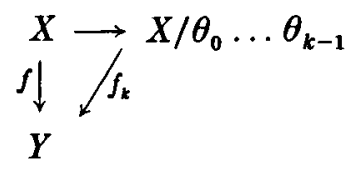

with $X / \theta_{0} \ldots \theta_{k-1} E(k-1)$ acyclic. Then by choosing (using (2-2a)) a $v_{k}$-self map of $X / \theta_{0} \ldots \theta_{k-1}$ we obtain a diagram

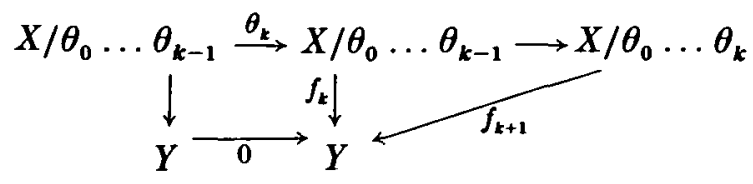

in which $X / \theta_{0} \ldots \theta_{k}$ is $E(k)$ acyclic. Eventually we obtain a morphism (in $\mathrm{C}_{n}(X)$ )

$$
\left(X / \theta_{0} \ldots \theta_{n-1}, f_{n}\right) \rightarrow(Y, f)
$$


Of course the objects $\left(X / \theta_{0} \ldots \theta_{n-1}, f\right)$ form a set, cofinal in $\mathrm{C}_{n}(X)$. We define $\mathrm{C}_{n}^{\prime}(X)$ to be the subcategory generated by these.

For $X=S^{0}$, the object $\left(S^{0} / \theta_{0} \ldots \theta_{n-1}, f\right)$ must realize a quotient map

$$
f_{*}: E(n)_{*} \rightarrow E(n)_{*}\left(S^{0} / \theta_{0} \ldots \theta_{n-1}\right)
$$

onto a cyclic torsion module (see [12]). Notice that as $r$ increases the smallest power of $I_{n}$ annihilating this cyclic module increases at least as rapidly as $r$. If we now set

$$
\begin{aligned}
\widetilde{E(n)} & =\underset{\widetilde{c_{n}\left(S^{0}\right)}}{\operatorname{holim}} E(n) \wedge Y \\
& =\underset{\widetilde{\mathrm{c}_{n}^{\prime}\left(S^{0}\right)}}{\operatorname{holim}} E(n) \wedge Y
\end{aligned}
$$

then $\widetilde{E(n)}$ is defined from a cofibre sequence

$$
\widetilde{E(n)} \rightarrow \prod_{\mathrm{C}_{n}^{\prime}\left(S^{0}\right)} E(n) \wedge Y \rightarrow \prod_{\mathrm{C}_{n}^{\prime}\left(S^{0}\right)} E(n) \wedge Y .
$$

But for each $Y$ in $\mathbf{C}_{n}^{\prime}\left(S^{0}\right)$

$$
\pi_{*}(E(n) \wedge Y) \cong E(n)_{*}(Y)
$$

is finitely generated over $E(n)_{*}$, and so for $s>0$,

$$
\underset{\mathrm{C}_{n}^{\prime}\left(S^{0}\right)}{\lim ^{s}} E(n) *(Y)=0
$$

by $(2 \cdot 2)$. Thus, using the spectral sequence

$$
E_{2}^{s^{*}}=\underset{\operatorname{Cim}_{n}^{s}\left(S^{0}\right)}{\lim ^{8}} E(n)_{*}(Y) \Rightarrow \pi_{*}(\widetilde{E(n))}
$$

we see that

$$
\pi_{*}(\widetilde{E(n)}) \cong \lim _{r} E(n)_{*} / I_{n}^{r}=\widehat{E(n)_{*}}
$$

Moreover the natural map $E(n) \rightarrow E(n) \wedge Y$ induces a (not obviously unique) morphism $E(n) \rightarrow \widetilde{E(n)}$. It is easily checked that such a map induces the canonical homomorphism $E(n)_{*} \rightarrow \widehat{E(n)}_{*}$ on homotopy.

$$
E(n) \rightarrow \widetilde{E(n)} \text { is a } K(n) \text { equivalence. }
$$

If $W$ is $K(n)$ acyclic, then $W \wedge D Y$ is $E(n)$ acyclic since it is $\mathrm{V}_{0 \leqslant j \leqslant n} K(j)$ acyclic. Hence as $E(n)$ is $E(n)$ local, we see that $E(n) \wedge Y$ is $K(n)$ local. By [2], 1.8 we have that $\widetilde{E(n)}$ is $K(n)$ local and

$$
E(n) \rightarrow \widetilde{E(n)} \text { is a } K(n) \text { localization. }
$$

There is a natural map

$$
E(n) \wedge Y \rightarrow \widehat{E(n)} \wedge Y
$$

and for $Y \in \mathrm{C}_{n}^{\prime}\left(S^{0}\right)$ we see that this is homotopy equivalence. So there is a compatible morphism $\widehat{E(n)} \rightarrow \overparen{E(n)}$ (again not obviously unique). As this induces a $K(n)$ equivalence and is a weak equivalence we deduce that

$$
\widehat{E(n)} \rightarrow \widetilde{E(n)}
$$


is an equivalence. Hence $E(n) \rightarrow \widehat{E(n)}$ is a $K(n)$ localization by $(4 \cdot 6)$. For $v_{n}^{-1} \mathrm{BP}$ the result follows by [2], 1.8 and our (2.5).

In fact, the spectrum $\overline{E(n)}$ can be seen to represent the cohomology theory (on $\left.\mathrm{CW}^{f}\right)$

$$
\widetilde{\widetilde{E(n)^{*}}}()=\underset{\lim _{n}\left(s^{0}\right)}{\operatorname{lin}}(E(n) \wedge Y)^{*}()
$$

using the methods of Section 2. Moreover, by $(2 \cdot 3)$ we see that the morphisms

$$
E(n) \rightarrow \widetilde{E(n)} \text { and } \widehat{E(n)} \rightarrow \widetilde{E(n)}
$$

are unique.

We remark that $L_{K(n)} E(n)$ can also be considered as $F\left(N_{n} S^{0}, E(n)\right)$ where $N_{n} S^{0}$ is the $n$th term in the Chromatic Tower for the sphere $S^{0}$ (see [15]). These results generalize those of Bousfield for the case $n=1$ (see [2]). A related discussion of $K(n)$ localization occurs in [5].

Unfortunately the natural map $v_{n}^{-1} \mathrm{BP} \rightarrow \widehat{v_{n}^{-1} \mathrm{BP}}$ is not a $K(n)$ equivalence, hence is not a $K(n)$ localization. However the following result was pointed out by M. Hopkins :

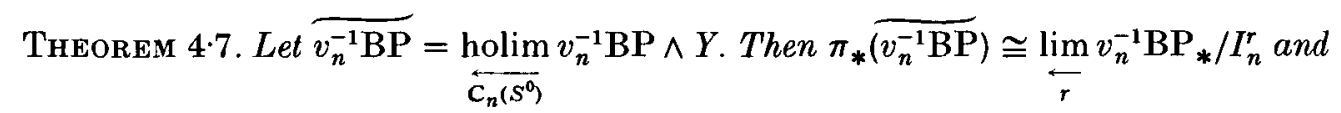

the natural map $v_{n}^{-1} \mathrm{BP} \rightarrow \widetilde{v_{n}^{-1} \mathrm{BP}}$ is a $K(n)$ localization.

The proof involves a modification of techniques already used in this section.

It seems plausible that $\widehat{v_{n}^{-1} \mathrm{BP}}$ splits as a product of suspensions of $\widehat{E(n)}$, although probably not multiplicatively. It would be interesting to characterize the Artinian completion $\widehat{v_{n}^{-1} \mathrm{BP}}$ in a way which is independent of the actual construction.

The first author would like to thank the SERC, Universität Bern and IHES for support whilst this work was in progress.

\section{REFERENCES}

[1] J. F. AdA ms. Stable Homotopy and Generalised Homotopy (University of Chicago Press, 1974).

[2] A. K. BousfieLd. The localization of spectra with respect to homology. Topology $18(1979)$, 257-287.

[3] M. J. Hopk INs. Global methods in homotopy theory. In Homotopy Theory, London Math. Soc. Lecture Note Ser. no. 117 (Cambridge University Press, 1987), pp. 73-96.

[4] C. U. Jensen. Les Foncteurs Dérivés de lim et leurs Applications en Théorie des Modules. Lecture Notes in Math. vol. 254 (Springer-Verlag, 1972).

[5] N. J. Kunn. Morava $K$-theories and infinite loop spaces. In Proceedings of the Arcata Conference (1986).

[6] P. S. LANDWEBER. BP $(\mathrm{BP})$ and typical formal groups. Osaka J. Math. 12 (1975), 357-363.

[7] P. Landweber. Homological properties of comodules over $\mathrm{MU}_{*} \mathrm{MU}$ and $\mathrm{BP} * \mathrm{BP}$. Amer. $J$. Math. 98 (1976), 591-610.

[8] J. Lubin and J. Tate. Formal moduli for one parameter formal Lie groups. Bull. Soc. Math. France 94 (1966), 49-60.

[9] H. Matsumura. Commutative Algebra (W. A. Benjamin, 1970).

[10] J. Morava. Noetherian localisations of categories of cobordism comodules. Ann. of Math. (2) 121 (1985), 1-39.

[11] H. R. Miller and D. C. Ravenel. Morava stabilizer algebras and the localisation of Novikov's $E_{2}$-term. Duke Math. J. 44 (1977), 433-447.

[12] M. R. Miller, D. C. Ravenel and W.S. Wilson. Periodic phenomena in the AdamsNovikov spectral sequence. Ann. of Math. (2) 106 (1977), 469-516. 
[13] D. C. Ravenel. Complex Cobordism and Stable Homotopy Groups of Spheres (Academic Press, 1986).

[14] D. C. Ravenel. Localisation with respect to certain periodic cohomology theories. $A m e r . J$. Math. 106 (1984), 351-414.

[15] D. C. Raven El. A geometric realisation of the chromatic resolution. In Proceedings of the J. C. Moore Conference (Princeton University Press, 1983).

[16] D. Sullivan. Genetics of homotopy theory. Ann. of Math. (2) 100 (1974), 1-79.

[17] U. WürgLer. A splitting for certain cohomology theories associated to BP*(-). Manuscripta Math. 29 (1979), 93-111.

[18] Z. Yosimura. On cohomology theories of infinite complexes. Publ. Res. Inst. Math. Sci. 8 $(1972-3), 295-310$. 